\title{
Sonographischer Zufallsbefund einer Lymphangioleiomyomatose (LAM)
}

Bei der Lymphangioleiomyomatose (LAM) handelt es sich um eine seltene gynäkotrope, idiopathische Erkrankung, die klinisch meist durch rezidivierende Pneumothoraces und chylösen Aszites auffällt. Wir möchten im Folgenden über eine Patientin berichten, bei der der sonographische Abdomenbefund richtungsweisend war.

\section{Fallbeschreibung}

Bei der jetzt 36-jährigen, ansonsten unauffälligen Patientin war im Rahmen einer Routineuntersuchung eine zunehmende laborchemische Einschränkung der Nierenfunktion festgestellt worden. Sie stellte sich zur sonographischen Abdomenuntersuchung vor.

In dieser ließen sich beide Nieren als solche nicht mehr abgrenzen, da sie durch einen oder mehrere echoreiche Tumoren durchsetzt waren (Abb.1,2). Inter-aorto-kaval bzw. links paraaortal zeigten sich teils echofreie, teils echoarme Veränderungen, die zum Teil kompressibel waren, in der Farbdopplersonographie aber kein Flusssignal aufwiesen (Abb. 2).

Bei den Nierenbefunden war in erster Linie an multiple Angioleimyolipome zu denken, die fast ausschließlich bei der

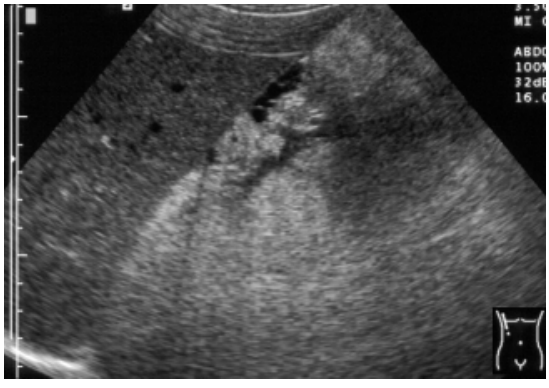

Abb.1 Längsschnitt über der rechten Niere. Die Niere lässt sich als solche nicht mehr abgrenzen, da sie von einem oder mehreren echoreichen Tumoren durchsetzt ist.

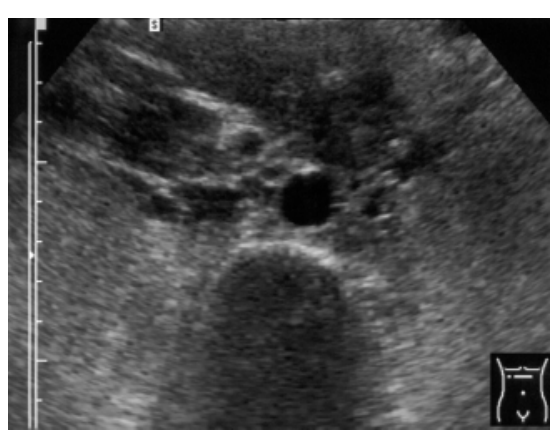

Abb. 2 Querschnitt knapp unterhalb des Abgangs der Nierenarterien aus der Aorta. Am Bildrand sind beidseits die Nieren miterfasst; in beiden finden sich echoreiche Tumoren. Inter-aorto-kaval bzw. links paraaortal zeigen sich teils echofreie, teils echoarme Veränderungen. 
tuberösen Sklerose, der Lymphangioleiomyomatose (LAM) sowie dem M. von-Hippel-Lindau vorkommen. Bei den Veränderungen inter-aorto-kaval bzw. links paraaortal war in Verbindung mit den Nierenbefunden insbesondere an zystisch veränderte Lymphknoten bzw. erweiterte Lymphgefäße zu denken.

Unter der Verdachtsdiagnose einer Lymphangioleimyomatose oder einer tuberösen Sklerose wurde eine computertomographische Untersuchung des Abdomens (Abb. 3) sowie des Thorax (Abb.4) durchgeführt, wegen der eingeschränkten Nierenfunktion allerdings nur nativ. Diese bestätigte zum einen die multiplen Angiomyolipome in beiden Nieren, die retroperitoneale Lymphadenopathie sowie die zystischen retroperitonealen Veränderungen. Zum anderen zeigte sie die typischen zystischen Lungenveränderungen einer Lymphangioleiomyomatose oder tuberösen Sklerose. Eine magnetresonanztomographische Untersuchung des Gehirns war (ebenso wie die klinische Inspektion) unauffällig, so dass es sich hier um eine Lymphangioleiomyomatose handelte.

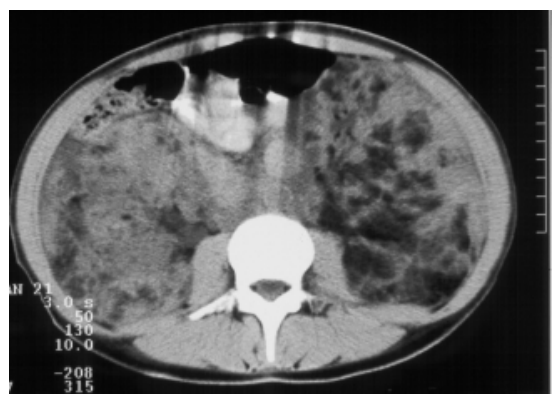

Abb. 3 Die native CT-Abdomenuntersuchung bestätigt die multiplen Angiomyolipome in beiden Nieren, die retroperitoneale Lymphadenopathie sowie die zystischen retroperitonealen Veränderungen.

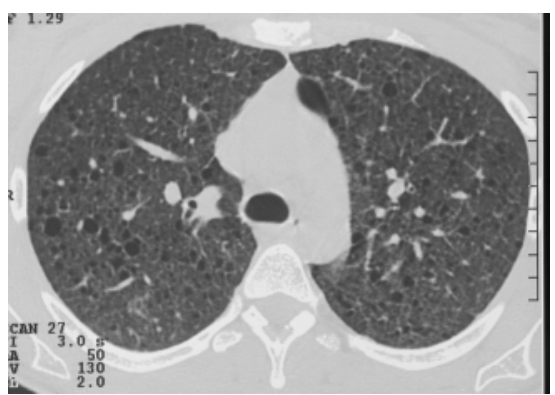

Abb. 4 Die CT-Thoraxuntersuchung zeigt multiple kleine zystische Lungenparenchymveränderungen.

\section{Diskussion}

Bei der Lymphangioleiomyomatose (LAM) handelt es sich um eine seltene idiopathische Erkrankung, die fast ausschließlich bei prämenopausalen Frauen vorkommt und durch die Proliferation abnormaler glatter Muskelzellen in der Lunge sowie im lymphatischen System von Thorax und Retroperitoneum charakterisiert ist.

Im konventionellen Thoraxbild finden sich als klassische Trias eine retikuläre interstitielle Zeichnungsvermehrung, ein chylöser Pleuraerguss sowie wiederholte Pneumothoraces (Aberle et al., Radiology 1990; 176: 381). Bei den interstitiellen Veränderungen in der konventionellen Thoraxübersichtsaufnahme handelt es sich um Überlagerungsfiguren dünnwandiger zystischer Veränderungen; diese stellen den wegweisenden Befund der Lymphangioleiomyomatose in der CT-Thoraxuntersuchung dar (Bernstein et al., Am J Respir Crit Care Med 1995; 152: 2138).

Die 4 häufigsten abdomino-pelvinen Befunde bei Patienten mit einer Lymphangioleiomyomatose umfassen Angiomyolipome der Nieren, eine Lymphadenopathie, Lymphangiome sowie chylösen Aszites (Maziak et al., Eur Respir J 1996; 9: 402, Woodring et al., Clin Imaging 1994; 18: 7).

Renale Angiomyolipome sind die häufigsten mit einer Lymphangioleiomyomatose assoziierten Tumoren (Maziak et al., Eur Respir J 1996; 9: 402). Mittels nativer CT-Untersuchung gelingt deren Diagnosestellung durch den Nachweis von Fett. Da es sich jedoch häufig um sehr heterogene Tumoren handelt, sind die fetthaltigen Anteile mitunter sehr klein und können im Einzelfall auch der Computertomographie entgehen. Die CT ist der Sonographie im Nachweis der Angiomyolipome überlegen, da sich diese sonographisch in etwa $1 / 4$ der Fälle isoechogen darstellen und so nicht vom Nierenparenchym zu differenzieren sind. Hauptkomplikation renaler Angiomyolipome ist die Blutung; Tumoren $>4 \mathrm{~cm}$ sollten daher halbjährlich mittels Sonographie oder CT kontrolliert werden. Symptomatische Angiomyolipome (Schmerzen, Hämaturie) können, wenn möglich, mittels partieller Nephrektomie oder Embolisation therapiert werden.
Lymphangioleiomyome entstehen durch die Proliferation glatter Muskelzellen in den Lymphgefäßen. Diese rufen eine Erweiterung und Obstruktion der Lymphgefäße mit resultierender zystischer Ansammlung chylösen Materials hervor.

Lymphangioleiomyome können zwischen den retroperitonealen vaskulären Strukturen liegen, diese im Einzelfall verlagern und mit einem malignen Prozess wie einem Lymphom verwechselt werden.

Bei etwa 40\% der Patientinnen mit einer Lymphangioleiomyomatose findet sich eine abdominelle Lymphadenopathie mit Lymphknoten bis zu $4 \mathrm{~cm}$ Größe. Diese Lymphknoten können sich hierbei sonographisch echofrei bzw. in der CT mit negativen Dichtewerten darstellen, da es sich hierbei um Fettansammlungen oder chylöse Lymphansammlungen (Lymphzysten) handeln kann. Die Aufweitung der Lymphzysten kann zu deren Ruptur mit Ausbildung chylösen Aszites führen.

Hauptdifferenzialdiagnose der Lymphangioleiomyomatose ist die tuberöse Sklerose (M. Bourneville-Pringle), wobei einige Literaturstellen angeben, dass es sich bei der Lymphangioleiomyomatose um eine „form fruste“ der tuberösen Sklerose handelt (Monteforte et al., Cancer $1974 ; 34: 317)$. Auch bei der tuberösen Sklerose finden sich multiple, meist bilaterale renale Angiomyolipome (bei $40-80 \%$ der Patienten) sowie bei den Frauen zystische Lungenveränderungen. Patienten mit einer Lymphangiomyomatose haben dagegen nicht die Veränderungen im Gehirn und die Hautveränderungen einer tuberösen Sklerose (kortikale Tubera, subependymale Knoten, retinale Hamartome, fasziale Angiofibrome oder periunguale Fibrome).

H. Strunk, W. Willinek, J. Textor, Bonn 